\title{
Major Characteristics and Functions of New Scheduling Software "Beeliner” Based on the Beeline Diagramming Method (BDM)
}

\author{
Seon-Gyoo Kim
}

\begin{abstract}
The construction environment has been changing rapidly over the last several years and with it the techniques used for managing projects. The schedule management technique, one of the construction management tools, has evolved as well in order to adapt to the increasingly complex construction environment. Most of the recently developed scheduling techniques are based on the Critical Path Method (CPM) that was proposed in 1956 and is classified into two categories; Arrow Diagramming (ADM) and Precedence Diagramming Methods (PDM). ADM is very good at graphically representing consecutive tasks, but it cannot express the relationships between two overlapping or concurrent activities. On the other hand, PDM can express the overlapping relationships but does not lend itself to visual representation. The growing complexity of the construction environment, in particular recently, increases the need for efficient schedule management and the ability to express the scheduling complexities introduced by tasks that must run at least in part concurrently. Most contractors prefer more the more visual format provided by the ADM method because it is more readily interpreted. Those software packages based on the PDM provide a means of addressing the overlapping tasks, however they do not have the ability to produce a graphical representation of the schedule that is desired by many contractors and managers. Beeliner is a new software product that is based on the Beeline Diagraming Method (BDM), which was originally proposed in 2010, developed into a product in 2012 and has the ability to express the overlapping/concurrent tasks while maintaining a readily understandable graphical representation of a schedule. This paper presents major features and applications of Beeliner, and provides construction professionals with an explanation of the new scheduling product and its applications.
\end{abstract}

Index Terms-Critical Path Method, Arrow Diagramming Method, Precedence Diagramming Method, Beeline Diagramming Method, Beeliner

\section{INTRODUCTION}

C RITICAL Path Method (CPM) has become increasingly more important in the construction industry over the last several decades. It integrates overall project management functions, such as scheduling, cost control, and resource planning and is therefore a critical part of schedule development. As the construction environment becomes increasingly more complex, the techniques for construction management have had to change accordingly.

The schedule management technique, one of the construction management tools, has evolved as well in order to adapt to the increasingly complex construction environment.
Most of the recently developed scheduling techniques are based on the Critical Path Method (CPM) that was proposed in 1956 and is classified into two categories; Arrow Diagramming (ADM) and Precedence Diagramming Methods (PDM). ADM is very good at graphically representing consecutive tasks, but it cannot represent the relationships between two overlapping or simultaneous activities. On the other hand, PDM can represent the relationships between two overlapping or simultaneous activities, but does not provide the graphical representation that is preferred by many contractors and managers. Recent changes in the complexity of the construction environment require an increased role of schedule management and the need to include the effects of tasks that run at least partially concurrently. This creates some tension between the efficacy of the PDM and the preference for a graphically represented schedule that may be provided by the ADM. This conflict between the need for representing overlapping or concurrent tasks and the desire for a readily interpreted graphical representation of the schedule emphasizes the need for a new product that has both capabilities.

Beeliner is a new software product that is based on the Beeline Diagraming Method (BDM), which was originally proposed in 2010, was developed into a product in 2012 and has the ability to express the overlapping/concurrent tasks while providing a readily interpreted the graphical representation of the schedule. This paper presents major features and applications of Beeliner (version 2.2), and provides construction professionals with an explanation of the new scheduling product and its applications.

\section{BeEline Diagramming Method}

\section{A. Basic Concept, Principle, and Characteristics}

The Beeline Diagramming Method (BDM) was proposed as a new networking technique by the author (2010). The basic concept of the BDM is to represent the overlapping relationship of two consecutive activities by the shortest straight line; this uses an arrow to represent the direction of workflow. The BDM connects any point of the predecessor to any point of the successor. This research defines the shortest straight line, which indicates a very direct or quick path or trip, as the "beeline” (Wiktionary 2009). The figure 1 shows the basic concept of the BDM; a beeline connects the middle point of the preceding activity $\mathrm{A}$ to the middle point of the succeeding 
activity B. The BDM has only one principle: The BDM represents the single or multiple overlapping relationships of two consecutive activities in the network by a beeline or beelines in any circumstance. Building on the basic concept and principle of the BDM, its characteristics are as follows.

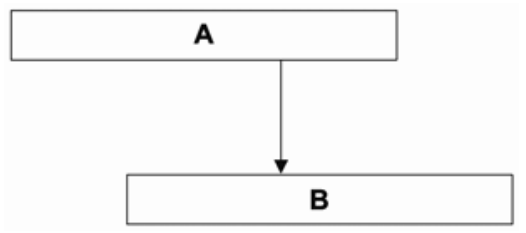

Fig. 1. Basic Concept of Beeline Diagramming Method

First, the BDM simplifies the overlapping relationships into one beeline. Thus, the complicated process of the Precedence Diagramming Method (PDM), which includes the positioning of activities, the selection of linkage types, and the calculation of the lead-time for the selected linkage, is eliminated.

Second, the BDM permits multiple overlapping relationships by means of multiple beelines between two consecutive activities. It therefore overcomes the limitations of the compound relationships found in the PDM, which has only two overlapping linkages.

\section{B. Linkage Representation Types in the BDM}

Linkage relationships between two consecutive activities in the BDM are represented differently from those in the PDM. Linkage relationships in the BDM can be represented at any middle point between two consecutive activities; the PDM, in contrast, represents linkage relationships only by FS, SS, FF, and SF relationships with lead-time between the starting and finishing points.

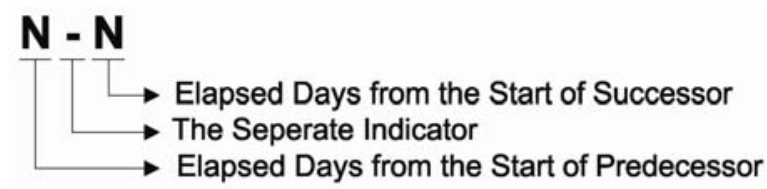

Fig. 2. Representation Type by the Elapsed Days

This research proposes three types of linkage representations in the BDM. The first is the "N-N" type shown in the figure 2. This type represents two consecutive activities that are mutually connected at any point in days after their respective starts.

The initial " $\mathrm{N}$ " in the figure 2 refers to the days that have elapsed from the start date of the preceding activity; the latter " $\mathrm{N}$ " refers to the days that have elapsed from the start date of the succeeding activity; the "-" is the separation indicator between the two Ns. An example of the first representation type is illustrated in the figure 3 . In this The figure, two consecutive activities that have inseparable work days are connected by a "7-4" type-between a point of 7 days after the start date of the preceding activity A and a point of 4 days after the start date of the succeeding activity B.

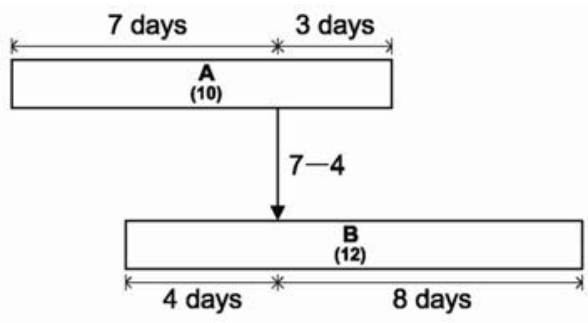

Fig. 3. An Example by the Elapsed Days

The second type is "N\%N", shown in the figure 4. This type represents two consecutive activities that are mutually connected after any percentage of the total duration of the two activities has elapsed.

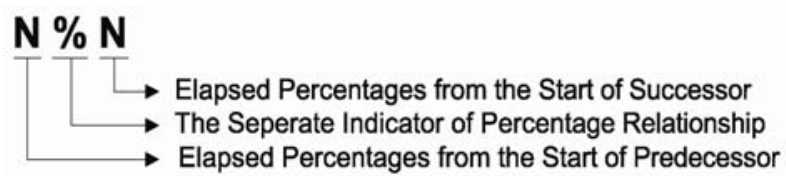

Fig. 4. Representation Type by the Elapsed Percentages

The initial " $N$ " in the figure 4 refers to the percentage of time elapsed since the start of the preceding activity and the latter " $N$ " refers to the percentage of time elapsed since the start of the succeeding activity; the "\%" is the separation indicator for the percentage relationship between the two Ns. An example of the second representation type is illustrated in the figure 5.

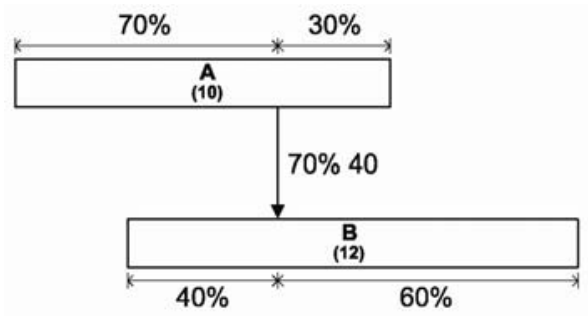

Fig. 5. An Example by the Elapsed Percentages

The two consecutive activities in the BDM are connected by a "70\%40" type-between a point where $70 \%$ of time has elapsed from the start date of the preceding activity A and a point where $40 \%$ of time has elapsed from the start date of the succeeding activity B.

The third is FS type, " $<\mathrm{N}>$ ”, shown in the figure 6, wherein the successor starts some days after the completion of the predecessor just like Finish-to-Start (FS) of PDM.

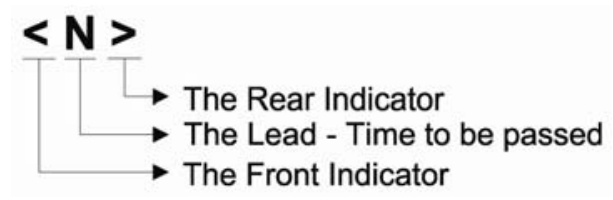

Fig. 6. Linkage Representation by FS Type

The " $N$ " in the figure 6 refers to the lead-time to be passed after the completion of the preceding activity. The initial " $<$ ” 
and latter ">" indicate the lead-time space indicators. An example of the FS representation type is illustrated in the figure 7.

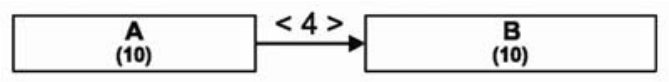

Fig. 7. An Example by FS Type

Two consecutive activities in the BDM are connected by “ $<4>$ ", wherein the succeeding activity B starts after the preceding activity A has been completed for 4 days.

The fourth type represents the multiple linkage relationships between two consecutive activities by the elapsed days or the second linkage type. Schedule computations will continue to be performed independently for each individual linkage. The figure 8 shows an example of the two-way multiple beeline relationships between two activities that have multiple milestones.

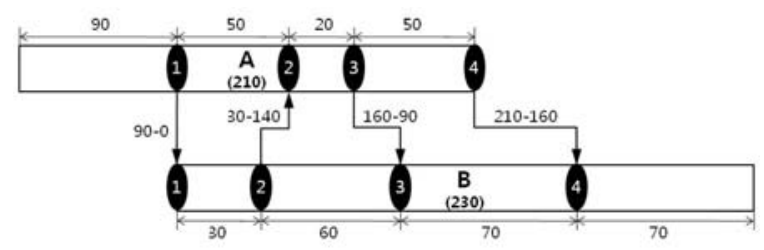

Fig. 8. Two-way Multiple Beeline Relationships

\section{INTRODUCTION OF BEELINER}

\section{A. Development Goals of Beeliner}

Innovation, practicality, and convenience are three important features that were built into the Beeliner product in order to provide an accurate and efficient means of managing construction schedules. The software embodies the latest advances in schedule management techniques as well as providing an interface that is intuitive and readily learned. The Beeliner product provides a significant advantage for project scheduling because it is based on the innovative and novel Beeline Diagramming Method (BDM), which is theoretically different than the other methods already used by the construction industry.

Existing scheduling software packages such as Primavera and MS-Project do not provide an efficient graphical representation of a PDM developed schedule. To address this, the software packages were made more complex by the addition of a large number of options that made the software difficult to use efficiently. An analysis of existing software packages and their users indicates that less than $10 \%$ of the existing software options are used on a regular basis, which suggests these features do not provide significant additional capabilities or assistance in practical schedule control, and may actually provide more confusion.

Steve Jobs, co-founder and former CEO of Apple, Inc., was a very strong advocate of simplicity in product design and function, and insisted that simple elegant solutions are more difficult to develop relative to complex ones, but provided better products. Using this underlying philosophy, the developers of Beeliner developed the software based on the "Beeline" concept which is simple in design and elegant in solution. This makes it more effective for project management and efficient for the users while reducing the complexity of the scheduling a project. Beeliner requires fewer options because its provides the means to solve problems in a more elegant fashion. As a result, Beeliner is much simpler to learn, use and implement in the field. One of the goals of the software development team was to design the interface so that its function is intuitive and seamless. The interface is menu driven and allows for ready maneuvering between functions such as moving, grouping and duplicating activities as well as providing a means of viewing the chart in a variety of scales, which is not possible in wither Primavera or MS-Project.

\section{B. Major Features of Beeliner}

Beeliner has a variety of unique features that are possible because the software is based on the Beeline principle, which is different than those utilized by the ADM and PDM. The major features of Beeliner are listed below;

1) Two-Screens Environment : Beeliner has the ability to display the grid and chart on two different monitors simultaneously. Existing scheduling software packages such as Primavera or MS-Project have a one-to-one connection between the data grid and the associated chart as shown in figure 9. As a result, they cannot show the entire grid or chart on the screen simultaneously. This requires the user to scroll to another portion of the grid and chart, which prevents the viewing the entire schedule in one frame.

The two-screen display is possible because Beeliner does not match the data grid with its associated chart on a one to one basis. This allows the grid and chart to be separated so that a user can perform a scheduling activity while viewing the resulting schedule chart. Beeliner has the ability to display all of the required function columns simultaneously as shown in figure 10.

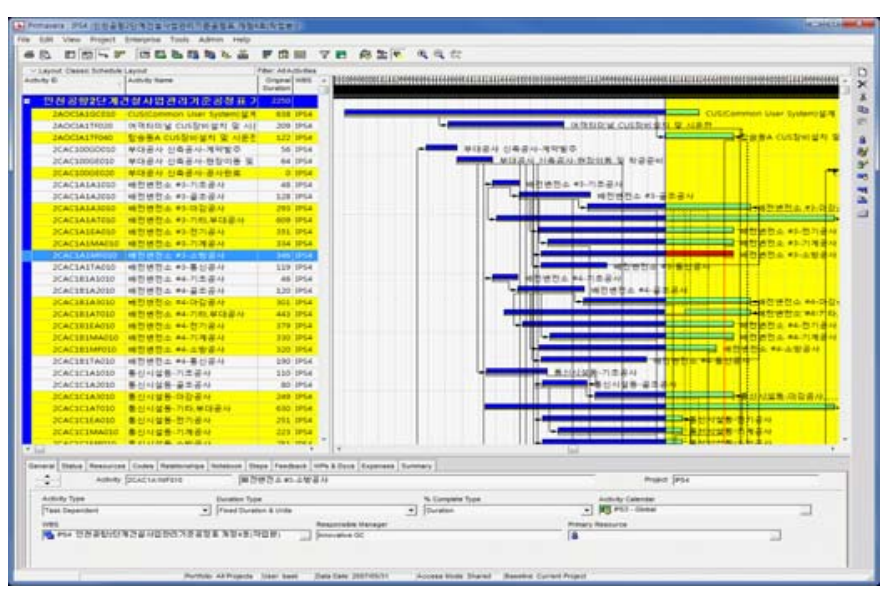


Fig. 9. Primvaera’s One Screen

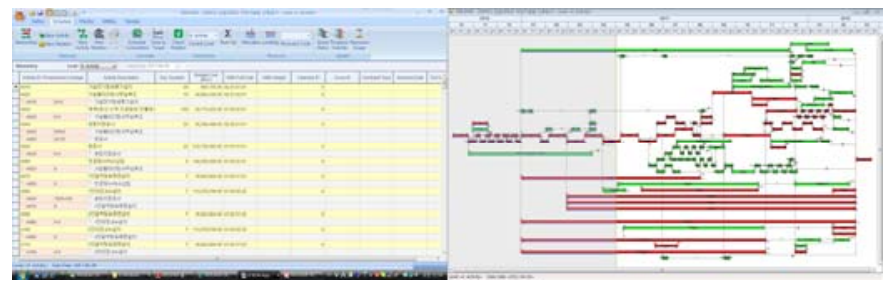

Fig.10. Beeliner's Two Screens

2) Automatic Activity Allocation: Beeliner places activities automatically on a chart using automatic allocation logic whenever a new activity is added. Other scheduling software does not provide this capability. Beeliner can add a task into a new row which can be placed below a previous activity. Figure 11 shows how automatic activity allocation works.

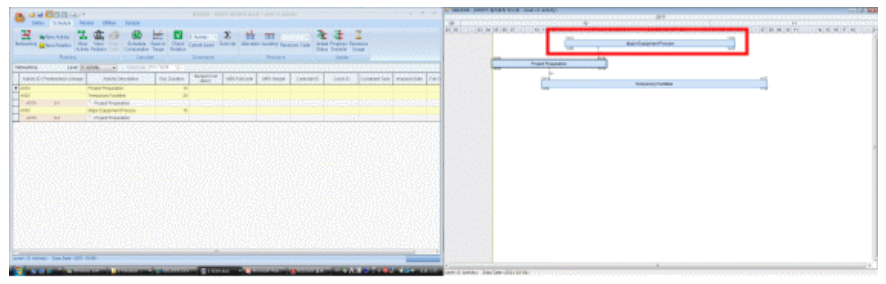

Fig. 11. Automatic Activity Allocation

Also, activities that was automatically allocated can be vertically moved to optimal location that user wants. The figure 12 shows activities to be vertically moved from the figure 11 .

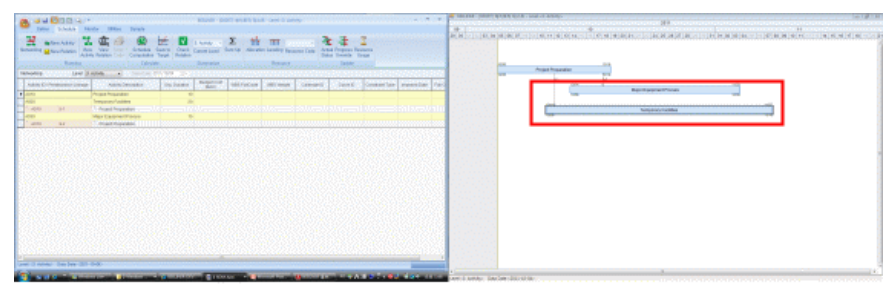

Fig. 12. Activity’s Vertical Movement

3) Enlarge \& Reduce of Chart: Beeliner provides a means of expanding and reducing the size of a chart by rotating the mouse wheel. This feature does not exist in either Primavera or MS-Project. This feature can help users check and review a specific area of a network clearly and in more detail. Beeliner can also display an entire CPM network on a single screen, as shown on figure 13, which demonstrates that Beeliner has both superior graphical capabilities (as provided by ADM software packages) that are desired by the construction industry and the ability to incorporate overlapping/concurrent tasks and relationships that are provided by systems based on the PDM. The overlapping relationships in Beeliner may be represented as the percentage of an overlap as well as two-way multiple overlapping.

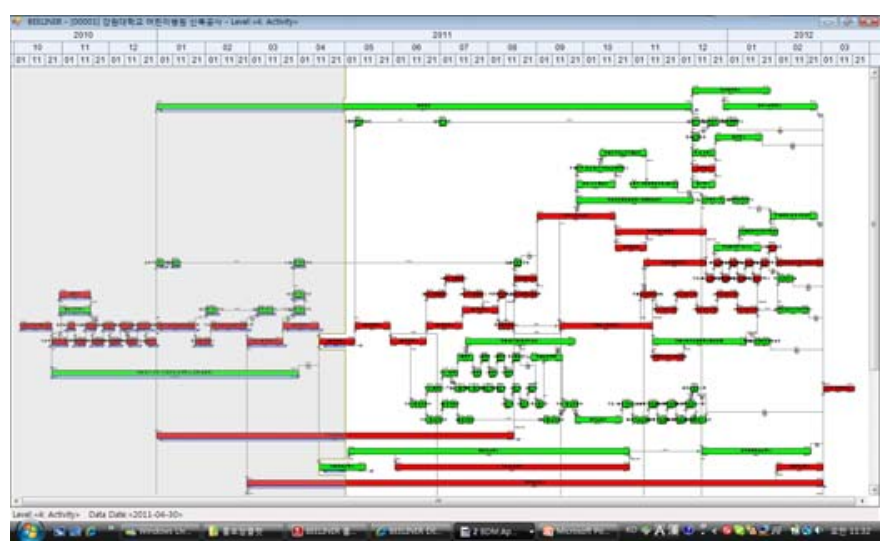

Fig. 13. A whole CPM network to be reduced into one chart screen

4) Duplicate of a Group of Activities in Chart: Beeliner provides a capability of moving and duplicating a group of activities in a chart by using the mouse wheel, which is not possible in either Primavera or MS-Project. This capability in Beeliner helps users develop a CPM network more efficiently.

5) Summarize CPM schedule: Beeliner can display all sub-schedules within a schedule hierarchy using the same CPM format by invoking the Sum-Up function as shown on figure 14. This allows all project participants (including high level managers and clients) to view and understand the entire project flow more clearly by viewing a summarized CPM schedule that has relationships between intermediate milestones of summarized activities. This function helps high level managers or clients make correct and effective decisions regarding the status of a project schedule.

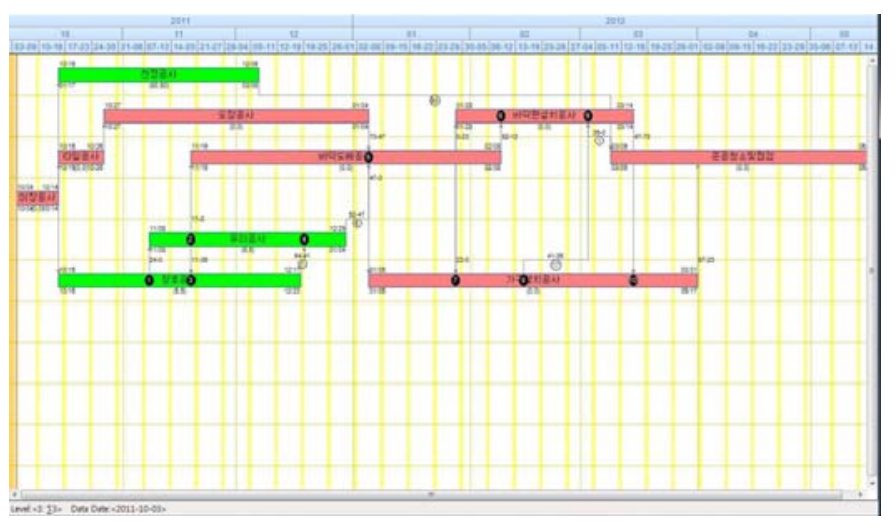

Fig. 14. Summarized CPM Schedule

6) Progress Override and Schedule Comparison: Figure 15 shows Progress Override and Schedule Comparison functions that can provide a means for a project team to determine the degree to which a project is delayed or ahead relative to a target schedule. This provides a means for the project team to develop an effective schedule recovery plan if necessary. 


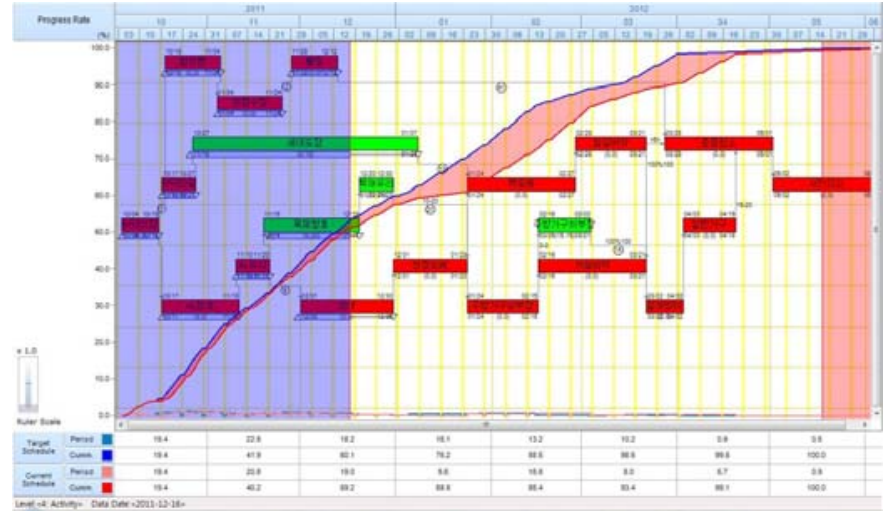

Fig. 15. Progress Override and Schedule Comparison

7) Integrate of Multiple Projects: Master Project is a function that is built into Beeliner that provides a means of integrating multiple sub-networks into a single master schedule. The Master Project function can integrate multiple summarized CPM schedules of a specific WBS level into one summarized CPM schedule. This helps a project team efficiently manage very large projects that have multiple sub-projects or components. Figure 16 shows an example of how multiple summarized CPM schedules are integrated into one summarized CPM schedule by the Master Project function.

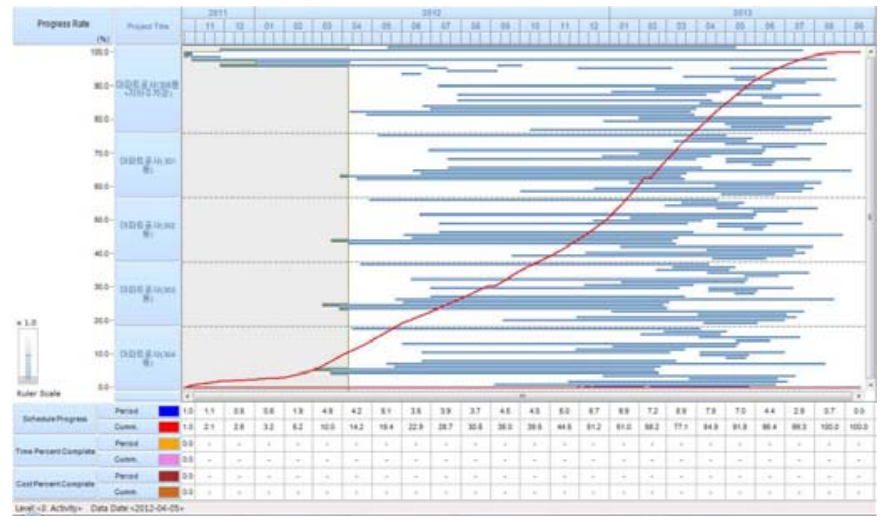

Fig. 16. Master Project

\section{Applications of BeELiner}

Beeliner provides a variety of benefits for improving schedule management procedures. The following are some of the most important benefits that a project management team may realize when using the Beeliner product.

\section{A. Verify CPM Schedule}

Beeliner can help verify a CPM schedule that was established by the PDM technique. A PDM network usually produces a significant number of unnecessary redundant relationships because the activities are listed vertically in the downward direction against the time axis and relationships between tasks are superimposed over each other like rain drops.

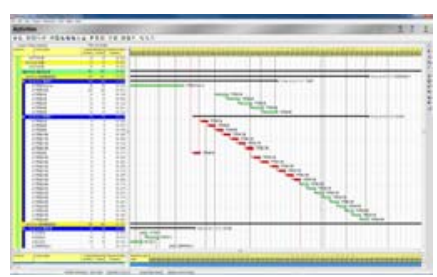

$<$ PDM schedule $>$

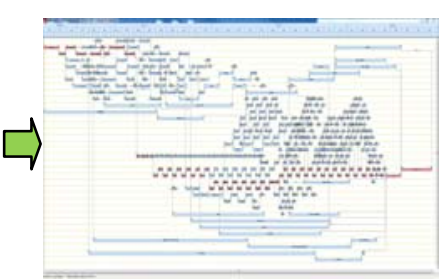

$<$ BDM schedule $>$

Fig. 17. Verify CPM Schedule

Beeliner produces almost no unnecessary relationships because activities and their corresponding relationships are represented in the horizontal direction with the time axis. Therefore, PDM networks can be verified by BDM networks by converting them to the MS-Excel format by using Input/Output functions of Beeliner in order to determine if the PDM network has the correct and necessary relationships. Unwanted or incorrect functions can then be eliminated or modified as needed. Steps of verifying a PDM schedule are as follows (refer to the figure 17);

(1) Convert a PDM to BDM schedule using MS-Excel by using the Input/Output functions of Beeliner.

(2) Review the suitability of the activities logic, and remove any unnecessary items.

\section{B. Summarize CPM Schedule for Top Managers}

Most summarized schedules produced by existing software packages represented using a bar chart format that cannot confirm relationships between summarized activities. If they can display the important relationships between major summarized activities, high level managers or clients can understand the status of a project more precisely, and then make more appropriate decisions regarding project schedule. The Sum-Up function summarizes the CPM schedule and in doing so provides an accurate status in order to provide decision making project participants with the necessary information to make project scheduling decisions. Steps of the Sum-Up function are as follows (refer to the figure 18);

(1) Summarize the CPM schedule beginning with the lower and then progressing to upper levels using the Sum-Up function of Beeliner.

(2) The upper level schedule shows a CPM schedule with two-way multiple overlapping relationships between intermediate milestones of summarized activities.

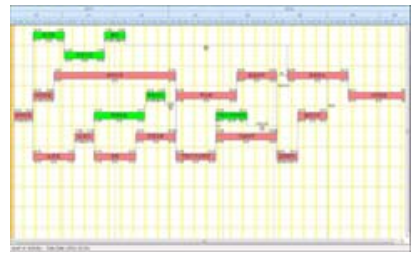

$<$ lower level schedule $>$

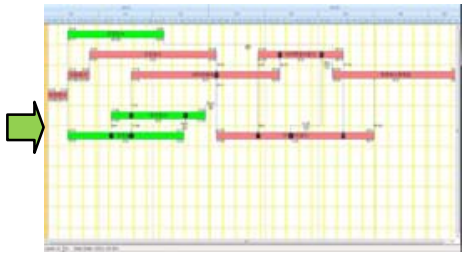

<upper level schedule>

Fig. 18. Summarizing CPM Schedule 


\section{Understand Activity Progress Clearly}

A project team or group of stakeholders can communicate schedule issues and make better and more informed scheduling decisions if they can recognize the progress of the various tasks and activities more clearly as provided by a clear graphical format. However, most existing scheduling software packages only represent the activity progress by comparing the plan with the actual status that is represented by a bar. If they have numerous activities, it is almost impossible for users to understand the overall project status as a whole.

Beeliner distinguishes the activity/task progress status by grouping them into regions of completed and uncompleted tasks based on the Data Date line that is provided in graphical format as shown in figure 19 . This provides users with a ready means of determining those activities that are complete and those that either are in progress or have yet to be started.

(1) The Data Date line changes shape to a staggered line when the actual progress of a task is inputted into the system.

(2) Left of the Data Date line is a region of completed activities and is indicated so by being colored. Right of Data Date line is the region of uncompleted activities and is indicated so by being as yet uncolored.

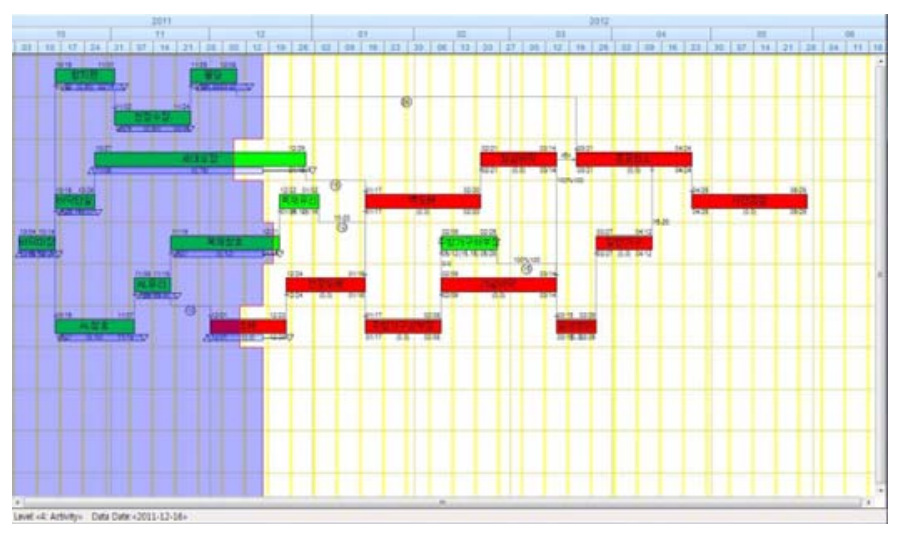

Fig. 19. Distinguish Progress Status

\section{Quantify Ahead or Delay of Schedule Accurately}

The Progress Override function of Beeliner helps a project team quantify more accurately the amount of time that the project is ahead or delayed based on current activities. Based on the result of Progress Override, a project team can establish an efficient schedule recovery plan. Steps of Progress Override are as follows (refer to figure 20);

(1) When executing Progress Override, those activities that are delayed are moved to the right and early ones to the left.

(2) Data Date line is converted from a staggered line to a straight one.

(3) If the last activity on a critical path is moved to the left, the status of the project is ahead of schedule. If it is moved to the right, the schedule is delayed with respect to the target.

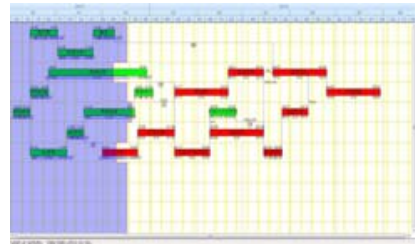

$<$ Before Progress Override $>$

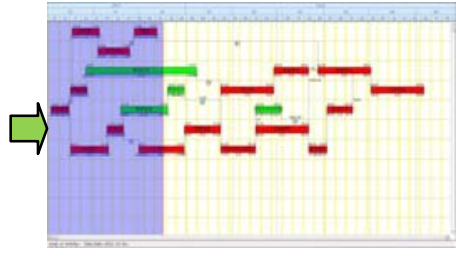

<After Progress Override>
Fig. 20. Progress Override

\section{E. Comprehend Schedule Differences Exactly}

The Schedule Comparison function helps a project team to precisely determine the difference between the target and current schedules in a graphical format. Based on the schedule comparison, a project team can determine whether a current schedule should be revised. Steps of Schedule Comparison are as follows (refer to figure 21);

(1) Select a target schedule and compare it to a current one.

(2) If there are deviations between the target and current schedules, they will expressed by gaps that will be colored red if the current project status is delayed relative to the target, and green color if current status is early relative to the target.

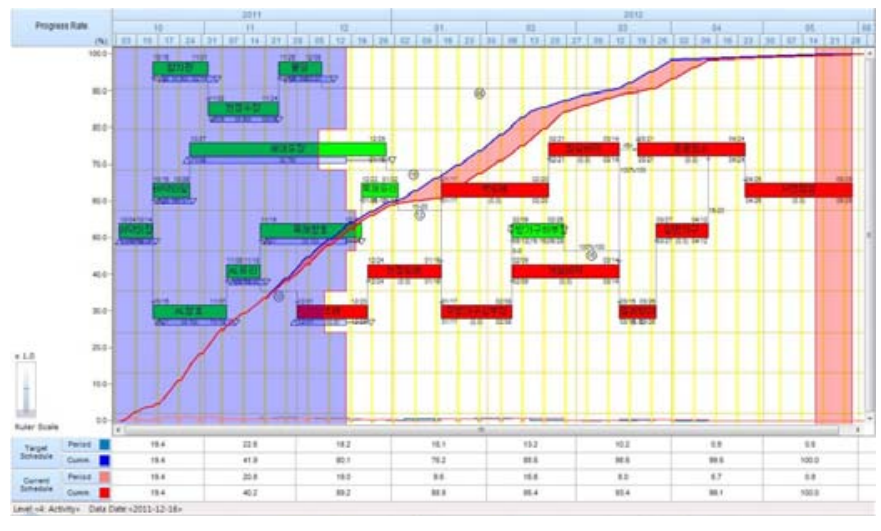

Fig. 21. Compare Schedules

\section{F. Manage a Big or Mega Project Effectively}

Mater Project function of Beeliner helps a project team manage very large and complex projects more efficiently. If a project is composed of multiple sub-projects, a project team establishes several CPM sub-networks at first, and then integrates them into one CPM schedule. This function provides an efficient means for a project team to control large and complex project projects that consist of multiple sub-networks. Steps of Master Project are as follows (refer to the figure 22);

(1) Establish multiple CPM sub-networks according to the components of a large project.

(2) Integrate multiple sub-networks into one CPM schedule by Master Project function, and then view the integrated plan and the actual project status as a whole. 


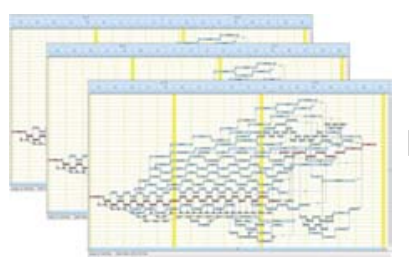

$<$ Multiple Sub-Networks $>$

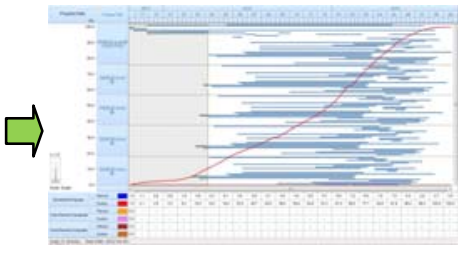

<Integrated Master Schedule>
Fig. 22. Integrating Multiple CPM Schedules

\section{G. Achieve Paperless Schedule}

Most companies are encouraging the use of electronic documents and paperless communication techniques to make storage and retrieval more efficient, to save money, and preserve natural resources. The ability for Beeliner to separate the Grid and Chart portions provides a means for each of them to be viewed independently so that a hard copy is not necessary in order to provide a client with a finished schedule without the data from which it is developed. This capability comes from the ability to view the Grid and Chart on two separate monitors as shown in figure 23;

(1) Separate Grid and Chart portions on two independent monitors.

(2) The Chart portion can be viewed using a high definition beam projector or a HDTV in an office or conference room as part of a presentation to management or clients.

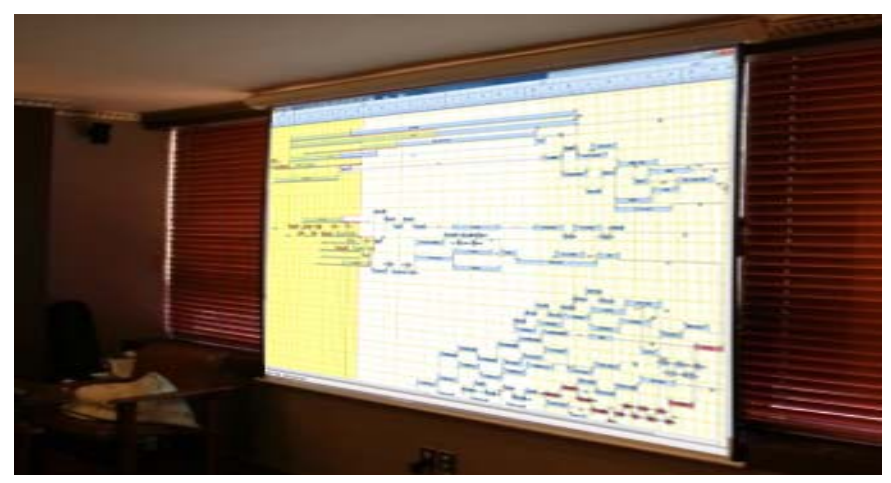

Fig. 23. Achieve Paperless Schedule

\section{CONCLUSIONS}

The recent and rapid changes experienced by the construction industry have made the role of schedule management increasingly important. The current scheduling techniques and tools have been challenged for a long time by the stringent requirements such as the need for flexible overlapping relationships and improved visual/graphical representation of the schedule. The current existing scheduling programs based on the PDM technique were proposed more than four decades ago and cannot satisfy these new requirements. Beeliner is a new scheduling software package that has been developed based the Beeline Diagramming Method (BDM) which was recently proposed in 2010 and is fundamentally different than those methods that were previously developed. Beeliner can utilize more flexible overlapping relationships of simultaneous or concurrent tasks and has the ability to provide superior visual/graphical representation of the schedule. This makes the Beeliner product simpler and more efficient to use in a dynamic and fast paced construction environment.

This paper presents the major features of Beeliner (version 2.2) which differentiate it from existing products. Features include; independent display of grid data and the resulting schedule, automatic activity allocation, chart size enlargement and reduction, activity duplication, CPM schedule summary, progress override and schedule comparison and multiple project integration. The Beeliner product also provides a means of verifying a CPM schedule and summarizing it for high level managers in order to provide a ready means of understanding the project status clearly and concisely. The software can clearly and accurately quantify the degree to which a project is ahead or behind schedule, explain schedule differences precisely, and provide a means to manage a large and/or complex project effectively while providing means of communicating the results electronically and without the need to use paper. Beeliner's innovative features and applications help a project team perform more effectively and enhance scheduling projects in the ever-changing project management environment. It successfully provides schedule guidance and confidence to management of all levels from the very beginning of a project up to the point of completion.

\section{REFERENCES}

[1] Kim, Seon-Gyoo, “Advanced Networking Technique”, Kimoondang, South Korea, 2010.

[2] Kim, Seon-Gyoo, “An Example of Representing Three Level's Schedules within Schedule Hierarchy by BDM Technique", Proceedings of the International Conference on Construction Engineering and Project Management, Sydney, Australia, 2011

[3] Kim, Seon-Gyoo, et al, "The Concept of Schedule and Cost Progress Integrated Management on the BDM network", Korean Journal of Construction Engineering and Management, November, Vo1 12(6), pp. 54-64, 2011.

[4] Kim, Seon-Gyoo, et al, "Schedule Computation Method of Two-way Multiple Overlapping Relationships on BDM Technique", Korean Journal of Construction Engineering and Management, March, Vo1 13(2), pp. 120-127, 2012.

[5] Kim, Seon-Gyoo, et al, "Method of Transforming PDM Overlapping Relationships to BDM Overlapping Relationships in CPM Schedule”, Korean Journal of Construction Engineering and Management, September, Vo1 13(5), pp. 144-152, 2012.

[6] Kim, Seon-Gyoo, "CPM Schedule Summarizing Function of the Beeline Diagramming Method", Journal of Asian Architecture and Building Engineering, November, Vol. 11 No. 2, pp. 367-374, 2012

Seon-Gyoo Kim. Ph.D, P.E., PMP, Professor of Architectural Engineering Department in College of Engineering at Kangwon National University, South Korea. He is a former vice president of Korean Institute of Construction Engineering and Management (KICEM), a member of ASCE. His areas of academic research interests include networking techniques, cost/schedule integration, and construction risk management. He has published many papers in various scientific journals and proceedings.. 